\title{
Development of scintillation detectors based on micro-pixel avalanche photodiodes
}

\section{Z. Sadygov}

Institute of Physics, AZ-1143 Baku, Azerbaijan, Joint Institute for Nuclear Research, 141980 Dubna, Russia

\section{F. Ahmadov $\mathbf{1}$, G. Ahmadov}

Institute of Radiation Problems, AZ-1143 Baku, Azerbaijan, Joint Institute for Nuclear Research, 141980 Dubna, Russia

E-mail: farid-akhmedov@yandex.ru

\section{R. Madatov}

Institute of Radiation Problems, AZ-1143 Baku, Azerbaijan

\section{E. Guliyev}

LLR Ecole Polytechnique, 91128 Palaiseau, France Cedex

\section{N. Anfimov, Z. Krumshtein, A. Olshevski, V. Shvetsov, V. Zhezher} Joint Institute for Nuclear Research, 141980 Dubna, Russia

$\mathrm{X}$. Abdullaev

National Academy of Aviation AZ1045, Baku, Azerbaijan

This article describes performance of a new generation of the Micro-Pixel Avalanche Photodiode (MAPD) with low capacitance. The gamma-ray and the alpha particle detecting performance of MAPD with LFS scintillator has been investigated using digital pulse processing techniques. The energy resolution was $11 \%$ and $6.5 \%$ for Cs-137(662keV) and Co-60(1.33MeV) gamma sources, respectively.

International Workshop on New Photon-detectors,

LAL Orsay, France

June 13-15, 2012 


\section{Introduction}

Micro Pixels Avalanche Photodiode (MAPD) has some distinguishing features, such as low operating voltage, high gain, high photon detection efficiency (PDE), insensitivity to magnetic field and compactness. All these features allow using MAPD instead of photomultiplier tubes in many applications [1-2].

It is known that scintillators are the most widely used in detectors for spectroscopy of energetic photons (X-rays and gamma rays). These detectors are commonly used in nuclear and high energy physics research, medical imaging, diffraction, nondestructive testing, nuclear treaty verification and safeguards, and geological exploration [3]. Last year MAPD with scintillators is also used widely in many applications: high energy physics (CALICE, ILC, NICA experiments) and civilian applications including free space (EUSO) and astronomy (MAGIC) and dosimetry and spectroscopy [4-7, 8]. Therefore, investigation of scintillation detectors based on MAPD gets more interesting.

\section{Mico-Pixel Avalanche Photodiode with low capacitance}

MAPD-3N1P consists of silicon substrate with n-type of conduction on the surface of which two $4 \mu \mathrm{m}$ deep epitaxial layers with the same specific resistance of $300 \Omega \cdot \mathrm{cm}$ and $7 \Omega \cdot \mathrm{cm}$ are grown. Special capacitance of these samples was much less previous generation [2] because of using high resistive epitaxial layer. An array of highly doped regions with n+-type of conduction with a step from 5 to $15 \mu \mathrm{m}$, depending on implementation, is formed between the epitaxial layers. The MAPD-3N1P samples were developed in collaboration with Zecotek Photonics Inc. Detail design and operation principle of the MAPD were described in $[1,8]$.

In Fig. 1 is shown capacitance-voltage characteristic of a sample named as MAPD$3 \mathrm{~N} 1 \mathrm{P}$ at room temperature. Dark current is $77 \mathrm{nA}$ around the operation voltage U 94V.

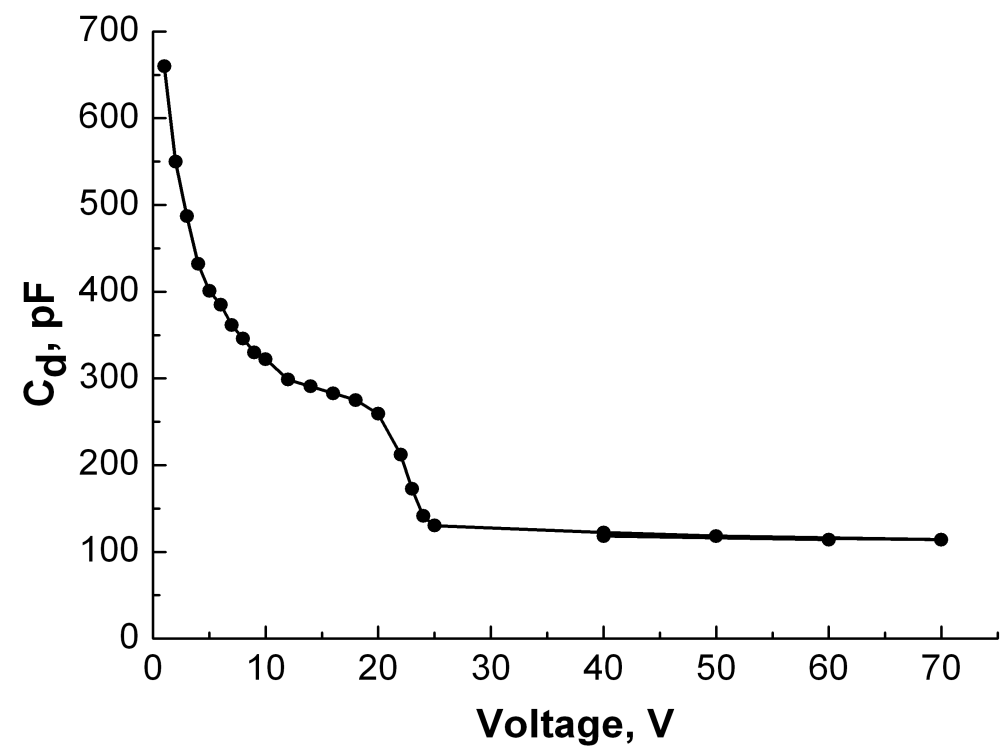

Figure 1: Capacitance-voltage characteristics of the MAPD-3N1P at room temperature. 
Capacitance of diode reaches $120 \mathrm{pF}$. Sensitive area of the MAPD-3N1P was $3 \mathrm{~mm} * 3 \mathrm{~mm}$. A blue light-emission-diode source having a low intensity was pulsed for short time duration (20 ns) to measure gain of diode. Gain of the diode reaches $5.5^{*} 10^{4}$ at room temperature. The maximum PDE is more than $30 \%$ around $430-525 \mathrm{~nm}$. However around $375 \mathrm{~nm}$ and $640 \mathrm{~nm}$ PDE is about $20 \%[6]$.

\section{Experimental details and discussion}

Gamma ray and alpha particle detecting studies were done on MAPD-3N1P samples consisting of 135000 pixels. The device gain can reach values of $5.5^{*} 10^{4}$ and photon detection efficiency is $25-30 \%$ for maximum emission wavelength of LFS (420-430nm) at operation voltage.

For detection gamma-ray and alpha particle were used LFS-3 and LFS-8 inorganic scintillators. Three different size LFS scintillators were used in the experiment (LFS-3: $2 \times 2 \times 10 \mathrm{~mm}^{3}$ and $2 \times 2 \times 20 \mathrm{~mm}^{3}$, LFS-8: $3 \times 3 \times 0.5 \mathrm{~mm}^{3}$ ). LFS-3 scintillator was used to detect gamma-rays from Cs- 137 and Co-60 sources. However, LFS-8 scintillator was used to detect gamma-rays and alpha particle from Sn-113 and Pu-238.

A block diagram of the experimental setup was described in Fig. 2. It consists of

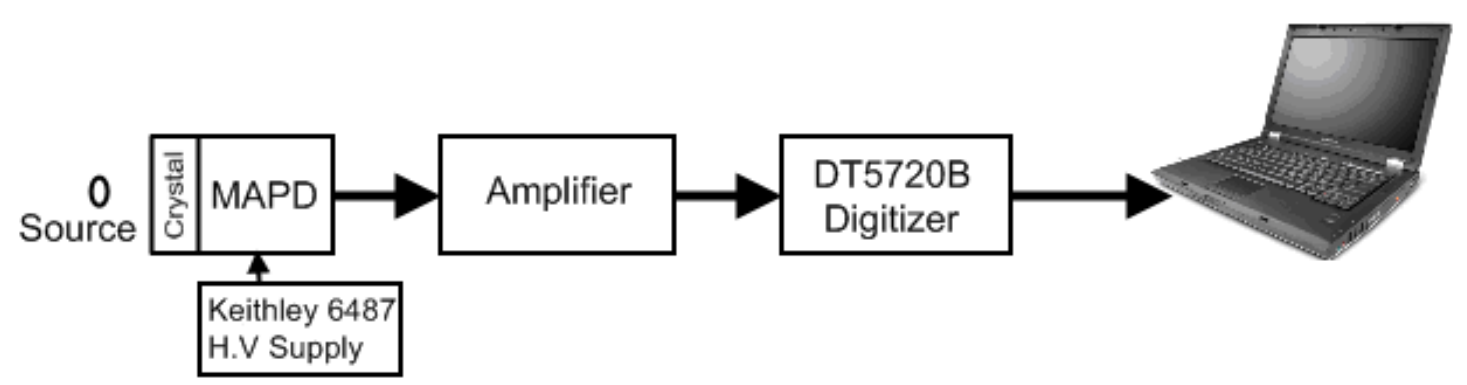

Figure 2: Block diagram of the experimental setup.

a scintillation crystal coupled to MAPD which is powered by Keithley 6487 high voltage source (H.V.), line amplifiers, LeCroy 2249A and a digitizer. The signal from MAPD is initially amplified by the line amplifier. The output of the liner amplifier is connected directly to LeCroy 2249A for detection gamma rays from Cs-137 and Co-60 gamma ray sources. However, pulse shape discrimination (PSD) was investigated 12 bits CAEN Desktop digitizer DT5720B, with 4 input channels. Each of the pulses is digitized with the sampling rate of $250 \mathrm{MS} / \mathrm{sec}$. The sampled data are read out by the personal computer through USB connection.

Peak positions and their full width at half maximum (FWHM) were obtained from Gaussian fit. The LFS-3 crystal was coated with aluminum tape and coupled to the MAPD with silicone optical grease.

$662 \mathrm{keV}$ and $1.33 \mathrm{MeV}$ gamma ray spectra measured with the LFS-3 scintillator were showed in Fig. 3. The measured energy resolutions for $662 \mathrm{keV}$ and $1.33 \mathrm{MeV}$ gamma ray energies were $11 \%$ and $6.5 \%$, respectively. 

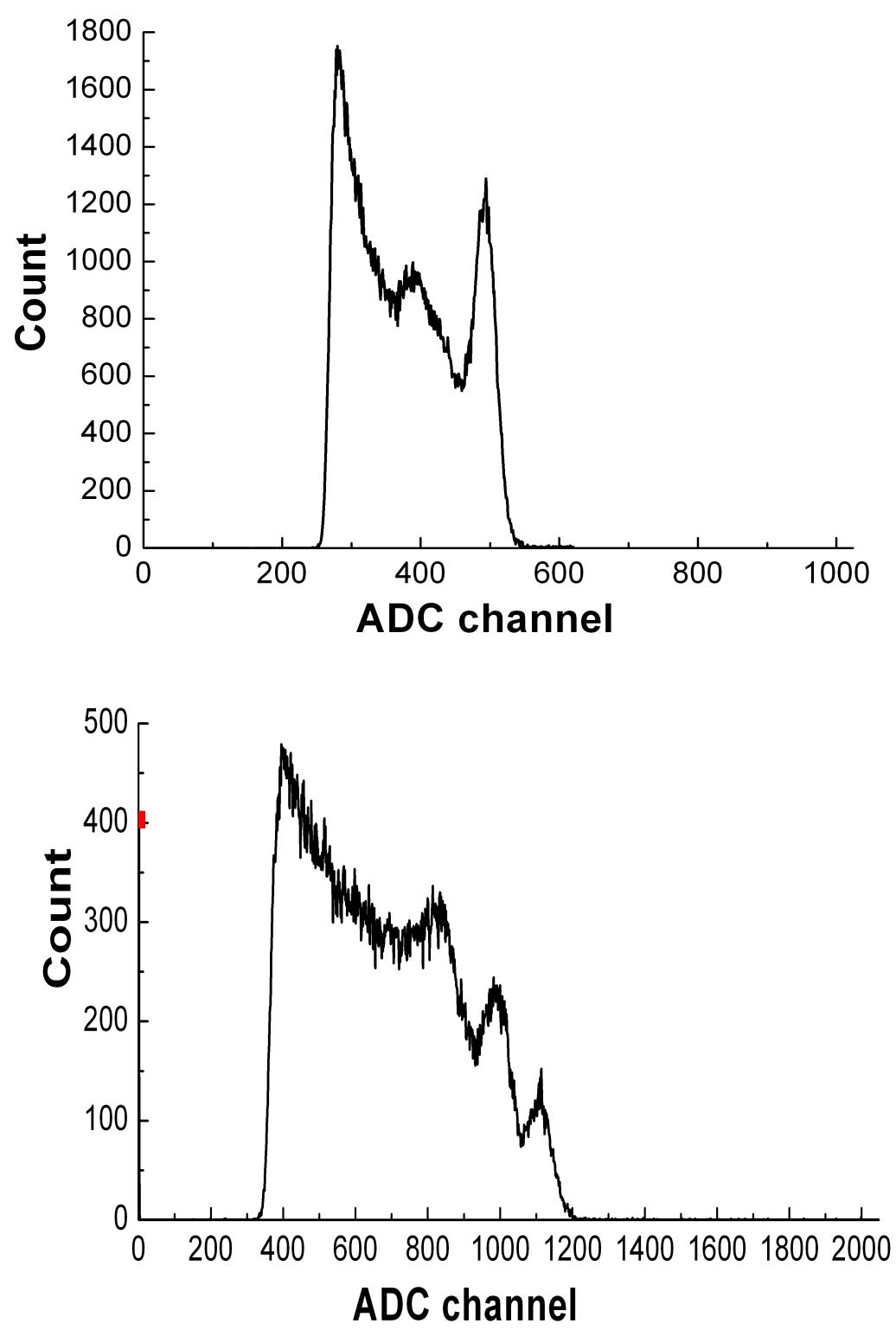

Figure 3: Amplitude spectra obtained at detection Cs-137(top) and Co-60(bottom) gamma rays with LFS- 3 crystal of size $2 * 2 * 10 \mathrm{~mm}^{3}$ and $2 * 2 * 20 \mathrm{~mm}^{3}$.

Alpha/gamma pulse shape discrimination was also investigated using of digital pulse processing techniques in LFS-8 scintillator with MAPD-3N1P in the experiment. The detector showed above was used as alpha and gamma detectors. Distance between LFS-8 crystal and alpha sources was $8 \mathrm{~mm}$. Alpha particles lost about $1 \mathrm{MeV}$ energy in air. As alpha and gamma source was used Pu-238(5.5MeV) and Sn-113 (391.7 keV).

A scatter plot of tail total ratio vs tail components of LFS-8 is showed in Fig. 4. Alpha particle pulse was sharper than gamma ray pulse and slow part of alpha particle pulse was far 
less than gamma ray. Therefore, this difference allows identifying alpha particle from gamma ray. The characteristic lines associated with alpha particle and gamma ray are clearly seen.

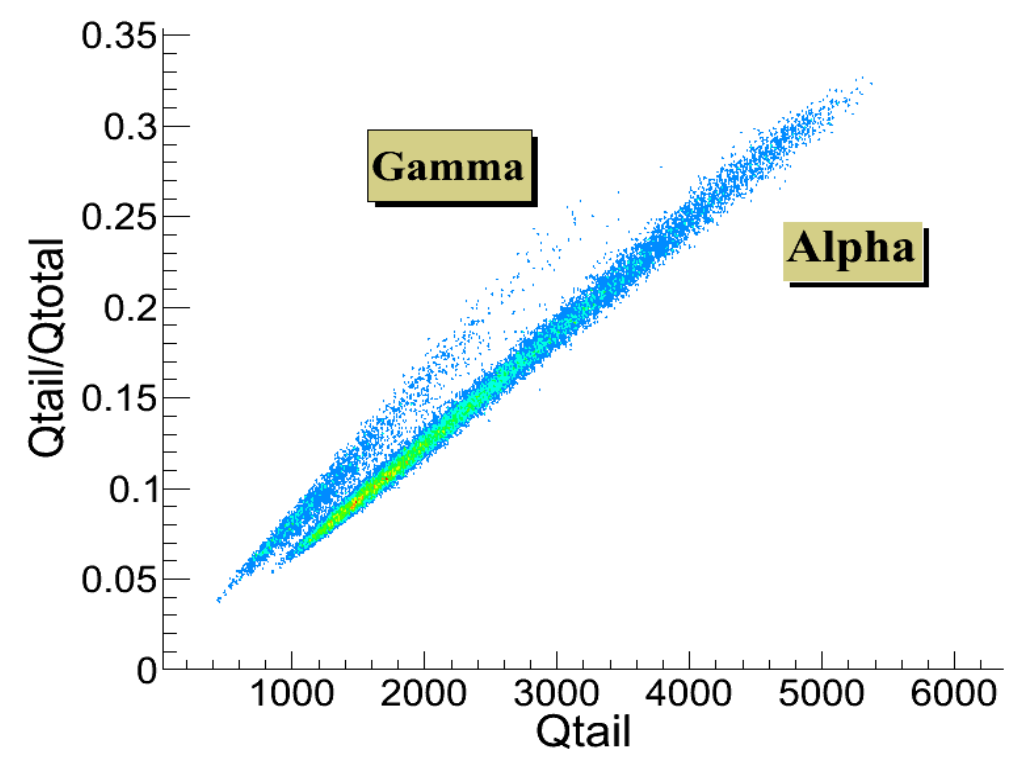

Figure 4: A scatter plot of tail total ratio vs tail components of LFS-8 for alpha particle from $\mathrm{Pu}-238$ and gamma ray from $\mathrm{Sn}-113$.

The obtained energy resolution for the $662 \mathrm{keV}$ and $1.33 \mathrm{MeV}$ gamma rays was $11 \%$ and $6.5 \%$. The results showed that MAPD together with LFS crystals $\left(2 \times 2 \times 10 \mathrm{~mm}^{3}\right)$, will be able to use successfully in the new generation of Positron Emission Tomography scanners.

PSD of LFS was investigated and characteristic lines of alpha particle and gamma ray were clearly seen. This performance allows using this kind of detectors for monitoring radioactive contamination in various environments and public security (Associated Particle Imaging for explosives and drugs detection) [7, 9].

\section{References}

[1] Sadygov Z. Ya., Russian Patent № 2316848, priority from 01.06.2006

[2] Z. Sadygov, A.F.Zerrouk, T.Bokova, A.Olshevski, N.Anfimov NIM-A 610 (2009) 390-392

[3] G. Knoll, Radiation Detection and Measurements $3^{\text {rd }}$ ed., John Wiley and Sons, 1999

[4] Y.Musienko et al, NIM-A, 610 (2009) 87-92

[5] F. Ahmadov, Z. Sadygov, R. Madatov, ICPPNE-2010, Baku, 08.10.2010

[6] Y. Musienko, NDIP-2011, Lyon, 08.07.2011 
[7] F. Ahmadov, G. Ahmadov, R. Madatov, Z. Sadygov, V. Zhezher, ISINN-19 (2011) 231-233

[8] http://www.zecotek.com

[9] V.M.Bystritski et al., Proceedings of the IV International Symposium on Technology and Mine Problem, Naval Postgraduate School, Monterey, California, March 13-16, 2000 